TOKYO J. MATH.

VoL. 3, No. 2, 1980

\title{
On the Wiener Formula of Functions of Two Variables
}

\author{
Kazuo ANZAI, Sumiyuki KOIZUMI and Katsuo MATSUOKA
}

\author{
Kagawa University and Keio University
}

\section{Introduction}

In dealing with the generalized harmonic analysis of functions of several variables, a problem of basic importance would be to study the so-called Wiener formula which states the equivalence of

$$
\lim _{s, T \rightarrow \infty} \frac{1}{4 S T} \int_{-T}^{T} \int_{-S}^{S}|f(s, t)|^{2} d s d t
$$

and

$$
\lim _{\varepsilon, \eta \rightarrow 0} \frac{1}{\pi^{2} \varepsilon \eta} \int_{-\infty}^{\infty} \int_{-\infty}^{\infty}|f(s, t)|^{2} \frac{\sin ^{2} \varepsilon s}{s^{2}} \frac{\sin ^{2} \eta t}{t^{2}} d s d t
$$

Different from the case of functions of one variable, one should keep in mind that there are several kinds of methods about limit processes and this makes the problem more involved than that of one variable. In this paper we restrict ourselves to the case of functions of two variables.

(1) The method of N. Wiener-A.C. Berry [9]. They assumed that the following limit

$$
\lim _{R \rightarrow \infty} \frac{1}{\pi R^{2}} \int_{0}^{2 \pi} \int_{0}^{R}|f(r \cos \theta, r \sin \theta)|^{2} r d r d \theta
$$

exists and developed the spectral analysis.

(2) The method of T. Kawata [1]. He assumed that the following limit

$$
\lim _{T, T^{\prime} \rightarrow \infty} \frac{1}{4 T T^{\prime}} \int_{-T^{\prime}}^{T^{\prime}} \int_{-T}^{T}|f(s, t)|^{2} d s d t
$$

exists and has the same limit no matter how $T$ and $T^{\prime}$ tend to infinity and, using the Wiener formula, derived some type of law of large num$\overline{\text { Received November }} 30,1979$ 
bers for nonstationary processes.

We begin with examining the Wiener formulas for several particular cases in the following section, and this indicates a new method of handling the problem. We shall deal with functions which satisfy that the limit

$$
\lim _{S, T \rightarrow \infty} \frac{1}{4 S T} \int_{-T}^{T} \int_{-S}^{S}|f(s, t)|^{2} d s d t
$$

exists and has the same limit for every positive constant $C$ whenever $S$ and $T$ tend to infinity in such a way that $S=C T$.

T. Kawata proved the Wiener formula directly, but it also follows from the general Tauberian theorem. See W. Rudin [8].

We first give a modification of the general Tauberian theorem and then establish the Wiener formula according to the method of $H$. R. Pitt [7].

We, here, give a remark. S. Koizumi [2] has studied about the spectral analysis of the Hilbert transform in the case of one variable. Recently K. Matsuoka $[4,5,6]$ has succeeded to extend the results to the case of functions of two variables using the Wiener formula we have obtained in this paper.

The authors are grateful to Professor T. Kawata for several valuable suggestions concerning the subject matter of this paper.

§1. Some examples.

1. Trigonometrical polynomial. Let us write

$$
p(x, y)=\sum_{n=0}^{N} \sum_{m=0}^{M} c_{m n} e^{i\left(\lambda_{m} x+\mu_{n} y\right)} .
$$

Then we easily see that

$$
\begin{aligned}
\frac{1}{4 S T} \int_{-T}^{T} & \int_{-S}^{S} p(x+s, y+t) \overline{p(s, t)} d s d t \\
\quad= & \sum_{n, l=0}^{N} \sum_{m, k=0}^{M} c_{m n} \overline{c_{k l}} e^{i\left(\lambda_{m} x+\mu_{n} y\right)} \frac{1}{2 S} \int_{-s}^{S} e^{i\left(\lambda_{m}-\lambda_{k}\right) s} d s \frac{1}{2 T} \int_{-T}^{T} e^{i\left(\mu_{n}-\mu_{l}\right) t} d t .
\end{aligned}
$$

Since

$$
\lim _{T \rightarrow \infty} \frac{1}{2 T} \int_{-T}^{T} e^{i \lambda t} d t= \begin{cases}1 & (\lambda=0) \\ 0 & (\lambda \neq 0),\end{cases}
$$


we have

$$
\begin{aligned}
\phi(x, y) & =\lim _{s, T \rightarrow \infty} \frac{1}{4 S T} \int_{-T}^{T} \int_{-S}^{s} p(x+s, y+t) \overline{p(s, t)} d s d t \\
& =\sum_{n=0}^{N} \sum_{m=0}^{M}\left|c_{m n}\right|^{2} e^{i\left(\lambda_{m} x+\mu_{n} y\right)}
\end{aligned}
$$

where $S$ and $T$ tend to infinity independently. Furthermore if we put

$$
\Lambda(u, v)=2 \pi \sum_{n: \mu_{n}<v} \sum_{m: \lambda_{m}<u}\left|c_{m n}\right|^{2}
$$

then

$$
\phi(x, y)=\frac{1}{2 \pi} \int_{-\infty}^{\infty} \int_{-\infty}^{\infty} e^{i(u x+v y)} d \Lambda(u, v)
$$

This is called the spectral representation of the correlation function of $p(x, y)$. In particular, we have, letting $x=y=0$,

$$
\lim _{S, T \rightarrow \infty} \frac{1}{4 S T} \int_{-T}^{T} \int_{-S}^{S}|p(s, t)|^{2} d s d t=\sum_{n=0}^{N} \sum_{m=0}^{M}\left|c_{m n}\right|^{2}
$$

On the other hand,

$$
\begin{aligned}
& \frac{1}{\pi^{2} \varepsilon \eta} \int_{-\infty}^{\infty} \int_{-\infty}^{\infty}|p(s, t)|^{2} \frac{\sin ^{2} \varepsilon s}{s^{2}} \frac{\sin ^{2} \eta t}{t^{2}} d s d t \\
& \quad=\sum_{n, l=0}^{N} \sum_{m, k=0}^{M} c_{m n} \frac{-1}{c_{k l}} \frac{1}{\pi \varepsilon} \int_{-\infty}^{\infty} \frac{\sin ^{2} \varepsilon s}{s^{2}} e^{i\left(\lambda_{m}-\lambda_{k}\right) s} d s \frac{1}{\pi \eta} \int_{-\infty}^{\infty} \frac{\sin ^{2} \eta t}{t^{2}} e^{i\left(\mu_{n}-\mu_{l}\right) t} d t,
\end{aligned}
$$

which yields, because of

$$
\begin{gathered}
\lim _{a \rightarrow 0} \frac{1}{\pi} \int_{-\infty}^{\infty} \frac{1-\cos a u}{a u^{2}} e^{i x u} d u= \begin{cases}1 & (x=0) \\
0 & (x \neq 0),\end{cases} \\
\lim _{\varepsilon, \eta \rightarrow 0} \frac{1}{\pi^{2} \varepsilon \eta} \int_{-\infty}^{\infty} \int_{-\infty}^{\infty}|p(s, t)|^{2} \frac{\sin ^{2} \varepsilon s}{s^{2}} \frac{\sin ^{2} \eta t}{t^{2}} d s d t=\sum_{n=0}^{N} \sum_{m=0}^{M}\left|c_{m n}\right|^{2},
\end{gathered}
$$

where $\varepsilon, \eta$ tend to 0 independently. Therefore we have the Wiener formula

$$
\begin{aligned}
\lim _{s, T \rightarrow \infty} & \frac{1}{4 S T} \int_{-T}^{T} \int_{-S}^{S}|p(s, t)|^{2} d s d t \\
& =\lim _{\varepsilon, \eta \rightarrow 0} \frac{1}{\pi^{2} \varepsilon \eta} \int_{-\infty}^{\infty} \int_{-\infty}^{\infty}|p(s, t)|^{2} \frac{\sin ^{2} \varepsilon s}{s^{2}} \frac{\sin ^{2} \eta t}{t^{2}} d s d t
\end{aligned}
$$

Similarly, if $(\lambda, \mu) \neq(0,0)$, then writing $\lambda s+\mu t=r \rho \cos \theta$, we see 
that

$$
\begin{gathered}
\frac{1}{\pi R^{2}} \iint_{s^{2}+t^{2} \leqq R^{2}} e^{i(\lambda s+\mu t)} d s d t=\frac{1}{\pi R^{2}} \int_{0}^{R} \int_{0}^{2 \pi} e^{i r \rho \cos \theta} r d \theta d r \\
=\frac{2}{R^{2}} \int_{0}^{R} J_{0}(r \rho) r d r=O\left(R^{-1 / 2}\right) \quad \text { as } \quad R \rightarrow \infty .
\end{gathered}
$$

Thus we also have

$$
\begin{aligned}
& \lim _{R \rightarrow \infty} \frac{1}{\pi R^{2}} \iint_{\varepsilon^{2}+t^{2} \leqq R^{2}}|p(s, t)|^{2} d s d t=\sum_{n=0}^{N} \sum_{m=0}^{M}\left|c_{m n}\right|^{2} \\
& \quad=\lim _{\varepsilon, \eta \rightarrow 0} \frac{1}{\pi^{2} \varepsilon \eta} \int_{-\infty}^{\infty} \int_{-\infty}^{\infty}|p(s, t)|^{2} \frac{\sin ^{2} \varepsilon s}{s^{2}} \frac{\sin ^{2} \eta t}{t^{2}} d s d t .
\end{aligned}
$$

2. A function with random signs. Let $(\lambda, \mu)$ be a pair of numbers in $(0,1) \times(0,1)$, with the binary expansion

$$
\begin{array}{ll}
\lambda=0 . \alpha_{11} \alpha_{12} \alpha_{21} \cdots(2), & \alpha_{i j}=0 \text { or } 1, \\
\mu=0 . \beta_{11} \beta_{12} \beta_{21} \cdots(2), & \beta_{k l}=0 \text { or } 1 .
\end{array}
$$

We define the function $f(x, y)$ as follows:

$$
f(x, y)= \begin{cases}2 \alpha_{2 m+1 n+1} & (m<x \leqq m+1, n<y \leqq n+1) \\ 2 \beta_{m+12 n+2} & (m<x \leqq m+1,-n-1<y \leqq-n) \\ 2 \beta_{m+12 n+1} & (-m-1<x \leqq-m, n<y \leqq n+1) \\ 2 \alpha_{2 m+2 n+1} & (-m-1<x \leqq-m,-n-1<y \leqq-n)\end{cases}
$$

$(m, n=0,1,2, \cdots)$. We wish to show that

$$
\begin{aligned}
\phi(x, y) & =\lim _{S, T \rightarrow \infty} \frac{1}{4 S T} \int_{-T}^{T} \int_{-S}^{S} f(x+s, y+t) \overline{f(s, t)} d s d t \\
& = \begin{cases}(1-|x|)(1-|y|) & (|x| \leqq 1,|y| \leqq 1) \\
0 & \text { (elsewhere), }\end{cases}
\end{aligned}
$$

where $S$ and $T$ tend to infinity under the restriction $S=C T$ for every positive constant $C$.

What we are going to show, may be stated also as follows: If $f(x, y)$ has over each square $(m, m+1) \times(n, n+1)(m, n=0, \pm 1, \pm 2, \cdots)$ either the value +1 or the value -1 , and if each choice of these values is independent of all the others, then the probability that $\phi(x, y)$ will not have the value given in (1.8) is 0 .

To begin with, let us note that for $m^{\prime} \leqq x<m^{\prime}+1, n^{\prime} \leqq y<n^{\prime}+1$, $\left|m^{\prime}\right|<M,\left|n^{\prime}\right|<N, M, N$ being large positive integers, we have 


$$
\begin{aligned}
& \frac{1}{4 M N} \int_{-N}^{N} \int_{-M}^{M} f(x+s, y+t) f(s, t) d s d t \\
& \quad=\frac{1}{4 M N} \sum_{n=-N}^{N-1} \sum_{m=-M}^{M-1}\left\{\iint_{(1)}+\iint_{(2)}+\iint_{(3)}+\iint_{(4)}\right\}\left({ }^{\prime \prime}\right) d s d t, \text { say , }
\end{aligned}
$$

where

$$
\begin{aligned}
\iint_{(1)}\left({ }^{\prime \prime}\right) d s d t & =\int_{n}^{n+n^{\prime}+1-y} \int_{m}^{m+m^{\prime}+1-x} f\left(m+m^{\prime}+1, n+n^{\prime}+1\right) f(m+1, n+1) d s d t \\
& =\left(m^{\prime}+1-x\right)\left(n^{\prime}+1-y\right) f\left(m+m^{\prime}+1, n+n^{\prime}+1\right) f(m+1, n+1), \\
\iint_{(2)}\left({ }^{\prime \prime}\right) d s d t & =\int_{n+n^{\prime}+1-y}^{n+1} \int_{m}^{m+m^{\prime}+1-x} f\left(m+m^{\prime}+1, n+n^{\prime}+2\right) f(m+1, n+1) d s d t \\
& =\left(m^{\prime}+1-x\right)\left(y-n^{\prime}\right) f\left(m+m^{\prime}+1, n+n^{\prime}+2\right) f(m+1, n+1), \\
\iint_{(3)}\left({ }^{\prime \prime}\right) d s d t & =\int_{n}^{n+n^{\prime}+1-y} \int_{m+m^{\prime}+1-x}^{m+1} f\left(m+m^{\prime}+2, n+n^{\prime}+1\right) f(m+1, n+1) d s d t \\
& =\left(x-m^{\prime}\right)\left(n^{\prime}+1-y\right) f\left(m+m^{\prime}+2, n+n^{\prime}+1\right) f(m+1, n+1), \\
\left.\iint_{(4)}^{(\prime \prime}\right) d s d t & =\int_{n+n^{\prime}+1-y}^{n+1} \int_{m+m^{\prime}+1-x}^{m+1} f\left(m+m^{\prime}+2, n+n^{\prime}+2\right) f(m+1, n+1) d s d t \\
& =\left(x-m^{\prime}\right)\left(y-n^{\prime}\right) f\left(m+m^{\prime}+2, n+n^{\prime}+2\right) f(m+1, n+1) .
\end{aligned}
$$

This representation gives us

$$
\begin{aligned}
\phi(x, y)= & \left(m^{\prime}+1-x\right)\left(n^{\prime}+1-y\right) \phi\left(m^{\prime}, n^{\prime}\right) \\
& +\left(m^{\prime}+1-x\right)\left(y-n^{\prime}\right) \phi\left(m^{\prime}, n^{\prime}+1\right) \\
& +\left(x-m^{\prime}\right)\left(n^{\prime}+1-y\right) \phi\left(m^{\prime}+1, n^{\prime}\right) \\
& +\left(x-m^{\prime}\right)\left(y-n^{\prime}\right) \phi\left(m^{\prime}+1, n^{\prime}+1\right) .
\end{aligned}
$$

Suppose we can prove that

$$
\phi(m, n)= \begin{cases}1 & ((m, n)=(0,0)) \\ 0 & (\text { elsewhere })\end{cases}
$$

Then we see that according as (i) $0<x \leqq 1,0<y \leqq 1$, (ii) $0<x \leqq 1,-1<y \leqq 0$, (iii) $-1<x \leqq 0,0<y \leqq 1$, and (iv) $-1<x \leqq 0,-1<y \leqq 0$, we have $\phi(x, y)=$ $(1-x)(1-y),(1-x)(1+y),(1+x)(1-y)$, and $(1+x)(1+y)$, and for other values of $(x, y), \phi(x, y)=0$, which can be written simply by

$$
\phi(x, y)= \begin{cases}(1-|x|)(1-|y|) & (|x| \leqq 1,|y| \leqq 1) \\ 0 & \text { (elsewhere) } .\end{cases}
$$

This, in turn, is written by 


$$
\phi(x, y)=\left(\frac{1}{2 \pi}\right)^{2} \int_{-\infty}^{\infty} \int_{-\infty}^{\infty}\left(\frac{\sin \frac{u}{2}}{\frac{u}{2}}\right)^{2}\left(\frac{\sin \frac{v}{2}}{\frac{v}{2}}\right)^{2} e^{i(u x+v y)} d u d v
$$

because of a well known property of Fejér integral. Now writing

$$
\Lambda(u, v)=\frac{1}{2 \pi} \int_{-\infty}^{v} \int_{-\infty}^{u}\left(\frac{\sin \frac{s}{2}}{\frac{s}{2}}\right)^{2}\left(\frac{\sin \frac{t}{2}}{\frac{t}{2}}\right)^{2} d s d t
$$

(1.11) can be represented by

$$
\phi(x, y)=\frac{1}{2 \pi} \int_{-\infty}^{\infty} \int_{-\infty}^{\infty} e^{i(u x+v y)} d \Lambda(u, v)
$$

Let us consider $f\left(m+m^{\prime}, n+n^{\prime}\right) f(m, n)$ for fixed $\left(m^{\prime}, n^{\prime}\right)$ and varying $(m, n)$. For any fixed $\left(m^{\prime}, n^{\prime}\right)$ each for them assumes either +1 or -1 for each $(m, n)$. Any set of signs are equal probably assigned to squares which are formed by any finite consecutive set of pairs $(m, n)$, and hence the each choise of any set of signs corresponds to the set of $(\lambda, \mu)$ of the same Lebesgue measure.

Let us put

$$
S_{M, N}\left(m^{\prime}, n^{\prime}\right)=\sum_{n=-N}^{N-1} \sum_{m=-M}^{M-1} f\left(m+m^{\prime}, n+n^{\prime}\right) f(m, n) .
$$

Then by an elementary consideration from the theory of probability, we have

$$
\begin{aligned}
& \left|\left\{(\lambda, \mu):\left|S_{M, N}\left(m^{\prime}, n^{\prime}\right)\right|>M N \varepsilon\right\}\right| \\
& \quad \leqq \frac{1}{2^{4 M N}} \sum_{k=[M N \varepsilon / 4]}^{M N} \frac{2 \cdot(4 M N) !}{(2 M N+2 k) !(2 M N-2 k) !} .
\end{aligned}
$$

By Stirling's theorem this is asymptotically

$$
\frac{2}{\sqrt{2 \pi}} \sum_{k=[M N \varepsilon / 4]}^{M N} e^{-4 k^{2} /(M N)}\left(\frac{1}{M N-\frac{k^{2}}{M N}}\right)^{1 / 2}=O\left((M N)^{1 / 2} e^{-M N \varepsilon^{2} / 4}\right)
$$

Now, take any pair of positive constants $C, D(C<D)$ and fix them. If we write $C=\tan \alpha, D=\tan \beta(0<\alpha<\beta<\pi / 2)$, then 


$$
\begin{aligned}
\sum_{(M, N): C \leqq N / M \leqq D}(M N)^{1 / 2} e^{-M N \varepsilon^{2} / 4} \\
\quad=O\left(\int_{1}^{\infty} \int_{\alpha}^{\beta} r(\sin \theta \cos \theta)^{1 / 2} e^{-\left(\varepsilon^{2} r^{2} \sin \theta \cos \theta\right) / 4} r d \theta d r\right) \\
\quad=O\left(\int_{1}^{\infty} r^{2} e^{-\left(\varepsilon^{2} \sin \alpha \cos \beta\right) / 4 \cdot r^{2}} d r\right)<\infty .
\end{aligned}
$$

The convergence of the above series implies from Borel-Cantelli lemma (transforming $(M, N)$ into single sequence) that the chance that

$$
\left|\sum_{n=-N}^{N-1} \sum_{m=-M}^{M-1} f\left(m+m^{\prime}, n+n^{\prime}\right) f(m, n)\right|>M N \varepsilon
$$

takes place infinitely often is zero. Hence, except for a set of pair of $(\lambda, \mu)$ of zero measure,

$$
\varlimsup_{M, N \rightarrow \infty}\left|\frac{1}{4 M N} \sum_{n=-N}^{N-1} \sum_{m=-M}^{M-1} f\left(m+m^{\prime}, n+n^{\prime}\right) f(m, n)\right| \leqq \frac{\varepsilon}{4},
$$

where $M, N$ tend to infinity under the restriction that $C \leqq N / M \leqq D$, for any pair of positive constants $C$ and $D$. Since $\varepsilon$ is arbitrarily, and the sum of a denumerable set of null sets is null, we should have

$$
\lim _{M, N \rightarrow \infty} \frac{1}{4 M N} \int_{-N}^{N} \int_{-M}^{M} f\left(m^{\prime}+s, n^{\prime}+t\right) f(s, t) d s d t=0,
$$

except for a null set of pair of values of $(\lambda, \mu)$, where $M, N$ tend to infinity under the restriction in consideration. The transition from integral $(M, N)$ to general values $(S, T)$ offers no difficulty, and we have

$$
\phi\left(m^{\prime}, n^{\prime}\right)=0 \quad\left(\left(m^{\prime}, n^{\prime}\right) \neq(0,0)\right),
$$

thus completing the proof of (1.9) and therefore (1.10).

3. Constant functions in a half-plane. Let us consider the function

$$
f(x, y)= \begin{cases}1 & (y \leqq x) \\ 0 & (y>x)\end{cases}
$$

Then for every $T>0$,

$$
\frac{1}{4 T^{2}} \int_{-T}^{T} \int_{-T}^{T}|f(s, t)|^{2} d s d t=\frac{1}{2}
$$

On the other hand for every $\varepsilon>0$, 


$$
\begin{aligned}
& \frac{1}{\pi^{2} \varepsilon^{2}} \int_{-\infty}^{\infty} \int_{-\infty}^{\infty}|f(s, t)|^{2} \frac{\sin ^{2} \varepsilon s}{s^{2}} \frac{\sin ^{2} \varepsilon t}{t^{2}} d s d t \\
& \quad=\frac{1}{2} \cdot \frac{1}{\pi^{2} \varepsilon^{2}} \int_{-\infty}^{\infty} \int_{-\infty}^{\infty} \frac{\sin ^{2} \varepsilon s}{8^{2}} \frac{\sin ^{2} \varepsilon t}{t^{2}} d s d t=\frac{1}{2} .
\end{aligned}
$$

Thus

$$
\begin{aligned}
\lim _{\substack{s, T \rightarrow \infty \\
s=T}} & \frac{1}{4 S T} \int_{-T}^{T} \int_{-S}^{S}|f(s, t)|^{2} d s d t \\
& =\lim _{\substack{\varepsilon, \eta \rightarrow 0 \\
\varepsilon=\eta}} \frac{1}{\pi^{2} \varepsilon \eta} \int_{-\infty}^{\infty} \int_{-\infty}^{\infty}|f(s, t)|^{2} \frac{\sin ^{2} \varepsilon s}{s^{2}} \frac{\sin ^{2} \eta t}{t^{2}} d s d t .
\end{aligned}
$$

While we see that

$$
\begin{gathered}
\lim _{T \rightarrow \infty} \frac{1}{4 T^{2}} \int_{-T}^{T} \int_{-T}^{T} f(x+s, y+t) f(s, t) d s d t \\
=\lim _{T \rightarrow \infty} \frac{\{2 T+(x-y)\}^{2}}{8 T^{2}}=\frac{1}{2} .
\end{gathered}
$$

Thus it can be represented by

$$
\begin{aligned}
\phi(x, y) & =\lim _{\substack{S, T \rightarrow \infty \\
S=T}} \frac{1}{4 S T} \int_{-T}^{T} \int_{-s}^{s} f(x+s, y+t) f(s, t) d s d t \\
& =\frac{1}{2 \pi} \int_{-\infty}^{\infty} \int_{-\infty}^{\infty} e^{i(u x+v y)} d \Lambda(u, v)
\end{aligned}
$$

where

$$
\Lambda(u, v)= \begin{cases}\pi & (u, v \geqq 0) \\ 0 & \text { (elsewhere) } .\end{cases}
$$

We give one more example. Let us consider the function

$$
f(x, y)= \begin{cases}1 & ((2 y-x)(2 y+x)<0) \\ 0 & (\text { elsewhere })\end{cases}
$$

Then for every $T>0$,

$$
\frac{1}{4 T^{2}} \int_{-r}^{T} \int_{-T}^{T}|f(s, t)|^{2} d s d t=\frac{1}{4}
$$

On the other hand for every $\varepsilon>0$,

$$
\frac{1}{\pi^{2} \varepsilon^{2}} \int_{-\infty}^{\infty} \int_{-\infty}^{\infty}|f(s, t)|^{2} \frac{\sin ^{2} \varepsilon s}{s^{2}} \frac{\sin ^{2} \varepsilon t}{t^{2}} d s d t
$$




$$
\begin{aligned}
& =\frac{4}{\pi^{2} \varepsilon^{2}} \int_{0}^{\infty}\left(\int_{0}^{s / 2} \frac{\sin ^{2} \varepsilon t}{t^{2}} d t\right) \frac{\sin ^{2} \varepsilon s}{s^{2}} d s \\
& =\frac{4}{\pi^{2} \varepsilon} \int_{0}^{\infty}\left(\int_{0}^{\varepsilon s / 2} \frac{\sin ^{2} v}{v^{2}} d v\right) \frac{\sin ^{2} \varepsilon s}{s^{2}} d s \\
& =\frac{4}{\pi^{2}} \int_{0}^{\infty}\left(\int_{0}^{u / 2} \frac{\sin ^{2} v}{v^{2}} d v\right) \frac{\sin ^{2} u}{u^{2}} d u .
\end{aligned}
$$

Since it is clear that for every $u>0$,

$$
\int_{0}^{u / 2} \frac{\sin ^{2} v}{v^{2}} d v>\int_{u / 2}^{u} \frac{\sin ^{2} v}{v^{2}} d v
$$

and

$$
\frac{4}{\pi^{2}} \int_{0}^{\infty}\left(\int_{0}^{u} \frac{\sin ^{2} v}{v^{2}} d v\right) \frac{\sin ^{2} u}{u^{2}} d u=\frac{1}{2},
$$

the above is strictly greater than $1 / 4$. That is

$$
\begin{aligned}
\lim _{\substack{s, T \rightarrow \infty \\
S=T}} & \frac{1}{4 S T} \int_{-T}^{T} \int_{-S}^{s}|f(s, t)|^{2} d s d t \\
& <\lim _{\substack{s, \eta \rightarrow 0 \\
\varepsilon=\eta}} \frac{1}{\pi^{2} \varepsilon \eta} \int_{-\infty}^{\infty} \int_{-\infty}^{\infty}|f(s, t)|^{2} \frac{\sin ^{2} \varepsilon s}{s^{2}} \frac{\sin ^{2} \eta t}{t^{2}} d s d t .
\end{aligned}
$$

Thus in this case, the Wiener formula fails to hold.

\section{§2. General Tauberian theorems.}

Let $G$ be a locally compact aberian group and $\gamma(x)$ be a character of $G$. The set of all continuous characters of $G$ forms a group $\Gamma$, the dual group of $G$.

If $f \in L^{1}(G)$, the convolution $(f * \phi)(x)$ may be regarded as an average of $\phi$ by assigning a weight factor $f(x-y)$ to the value $\phi(y)$. Then we have

TheOREM (N. Wiener). Suppose $\phi \in L^{\infty}(G), f \in L^{1}(G), \hat{f}(\gamma) \neq 0$ for all $\gamma \in \Gamma$, and

$$
(f * \phi)(x) \longrightarrow a \hat{f}(0) \quad(x \longrightarrow \infty)
$$

Then the limit relation

$$
(g * \phi)(x) \longrightarrow a \hat{g}(0) \quad(x \longrightarrow \infty)
$$

holds for every $g \in L^{1}(G)$.

If we impose slightly stronger conditions on $\phi$, the conclusion of the 
preceding theorem may be replaced by the stronger assertion that $\phi(x) \rightarrow a$ as $x \rightarrow \infty$.

Let us call a function $\phi \in L^{\infty}(G)$ slowly oscillating if $\phi(x)-\phi(y) \rightarrow 0$ as $x \rightarrow \infty$ and $x-y \rightarrow 0$. More explicitly, we require that for each $\varepsilon>0$, there should exist a compact set $K$ in $G$ and a compact neighborhood $V$ of 0 in $G$ such that $|\phi(x)-\phi(y)|<\varepsilon$ if $x-y \in V$ and $x \notin K$. Then we have

Theorem (H. R. Pitt). Suppose $\phi \in L^{\infty}(G), \phi$ is slowly oscillating, $f \in L^{1}(G), \hat{f}(\gamma) \neq 0$ for all $\gamma \in \Gamma$, and

$$
(f * \phi)(x) \longrightarrow a \hat{f}(0) \quad(x \longrightarrow \infty) .
$$

Then $\phi(x) \rightarrow a$ as $x \rightarrow \infty$.

For $f \in L^{1}(G)$, we define $Z(f)$ to be the set of all $\gamma \in \Gamma$ such that $\hat{f}(\gamma)=0$, and if $I$ is an ideal in $L^{1}(G)$, we define the zero-set of $I$ by

$$
Z(I)=\bigcap_{f \in I} Z(f) \text {. }
$$

Thus $\gamma \in Z(I)$ if and only if $\hat{f}(\gamma)=0$ for all $f \in I$. Let us notice that if $I$ is a closed ideal in $L^{1}(G)$ with $Z(I)$ empty, then $I=L^{1}(G)$. These theorems are based on this result.

Now let us modify these theorems to meet our purpose. Let us consider a path $x(t)$ in $G$ with a real parameter $t$ such that

$$
x(0)=0, x(t) \longrightarrow \infty \text { as } t \longrightarrow \infty \text {. }
$$

This means that for every compact set $K$ there exists a positive number $T$ such that $x(t)$ belongs to the complement of $K$ for all $t \geqq T$. Then we have

THEOREM 1. Suppose $\phi \in L^{\infty}(G), f \in L^{1}(G), \hat{f}(\gamma) \neq 0$ for all $\gamma \in \Gamma$, and

$$
(f * \phi)(x(t)-u) \longrightarrow a \hat{f}(0) \text { as } t \longrightarrow \infty(\forall u \in G) \text {. }
$$

Then the limit relation

$$
(g * \phi)(x(t)-u) \longrightarrow a \hat{g}(0) \text { as } t \longrightarrow \infty(\forall u \in G)
$$

holds for every $g \in L^{1}(G)$.

Proof. Replacing $\phi$ by $\phi-a$, we may assume, without loss of generality, that $a=0$. The set $I$ of all $g \in L^{1}(G)$ such that $(g * \phi)(x(t)-u) \rightarrow 0$ as $t \rightarrow \infty$ for all $u \in G$ is a linear subspace of $L^{1}(G)$ which is clearly translation-invariant. Also $I$ is closed, for if $g_{n} \in I$ and $\left\|g_{n}-g\right\|_{1} \rightarrow 0$, then $\left\|g_{n} * \phi-g * \phi\right\|_{\infty} \rightarrow 0$; and $g \in I$. Hence $I$ is a closed ideal in $L^{1}(G)$ with 
$Z(I)$ empty, and so $I=L^{1}(G)$.

Next let us call a function $\phi \in L^{\infty}(G)$ slowly oscillating along a path $x(t)$ if $\phi(x(t)-u)-\phi(y-u) \rightarrow 0$ as $t \rightarrow \infty$ and $x(t)-y \rightarrow 0$ for all $u \in G$. More explicitly, we require that for each $\varepsilon>0$, there should exist a positive number $T$ and a compact neighborhood $V$ of 0 in $G$ such that $|\phi(x(t)-u)-\phi(y-u)|<\varepsilon$ if $t \geqq T$ and $x(t)-y \in V$. We then have

THEOREM 2. Suppose $\phi \in L^{\infty}(G), \phi$ is slowly oscillating along a path $x(t), f \in L^{1}(G), \hat{f}(\gamma) \neq 0$ for all $\gamma \in \Gamma$, and

$$
(f * \phi)(x(t)-u) \longrightarrow a \hat{f}(0) \text { as } t \longrightarrow \infty(\forall u \in G) .
$$

Then $\phi(x(t)-u) \rightarrow a$ as $t \rightarrow \infty \quad(\forall u \in G)$.

Proof. Given $\varepsilon>0$, choose $T$ and $V$ as above, and let $g$ be the characteristic function of $V$, divided by $m(V)$. Then

$$
\phi(x(t)-u)-(g * \phi)(x(t)-u)=\frac{1}{m(V)} \int_{V}\{\phi(x(t)-u)-\phi(x(t)-u-y)\} d y,
$$

so that $|\phi(x(t)-u)-(g * \phi)(x(t)-u)|<\varepsilon$ for all $t \geqq T$ and $u \in G$. By Theorem 1 , $(g * \phi)(x(t)-u) \rightarrow a$ as $t \rightarrow \infty$ for all $u \in G$, and the desired conclusion follows.

We shall slightly generalize these theorems for later purposes. Let $L_{0}$ be a subclass of $L^{1}(G)$ such that for every $\gamma \in \Gamma$, there exists $f$ in $L_{0}$ with $\hat{f}(\gamma) \neq 0$. Then we have

TheOREM 1'. Suppose $\phi \in L^{\infty}(G)$, and for all $f \in L_{0}$,

$$
(f * \phi)(x(t)-u) \longrightarrow a \hat{f}(0) \text { as } t \longrightarrow \infty(\forall u \in G) .
$$

Then the limit relation

$$
(g * \phi)(x(t)-u) \longrightarrow a \hat{g}(0) \quad \text { as } \quad t \longrightarrow \infty \quad(\forall u \in G)
$$

holds for every $g \in L^{1}(G)$.

TheOREM 2'. Suppose $\phi \in L^{\infty}(G), \phi$ is slowly oscillating along a path $x(t)$, and for all $f \in L_{0}$,

$$
(f * \phi)(x(t)-u) \longrightarrow a \hat{f}(0) \text { as } t \longrightarrow \infty(\forall u \in G) .
$$

Then $\phi(x(t)-u) \rightarrow a$ as $t \rightarrow \infty \quad(\forall u \in G)$.

\section{$\S 3$. The Wiener formula.}

Let $R^{2}$ be the two-dimensional Euclidean space, $M\left(R^{2}\right)$ be a subclass 
of $L^{1}\left(R^{2}\right)$, consisting of all continuous functions $f$ for which

$$
\sum_{n=-\infty}^{\infty} \sum_{m=-\infty}^{\infty} \max _{\left(x_{1}, x_{2}\right) \in I_{m, n}}\left|f\left(x_{1}, x_{2}\right)\right|<\infty,
$$

where $I_{m, n}=\left\{\left(x_{1}, x_{2}\right): m \leqq x_{1} \leqq m+1, n \leqq x_{2} \leqq n+1\right\}$. Then for every $f \in$ $M\left(R^{2}\right)$, its Fourier transform $\hat{f}$ is defined for every pair of real arguments. Let us also introduce a subclass $M_{0}$ of $M\left(R^{2}\right)$, consisting of $f$ which satisfies the following property:

$$
\forall\left(u_{1}, u_{2}\right) \in R^{2}, \quad \exists f \in M_{0}: \hat{f}\left(u_{1}, u_{2}\right) \neq 0 .
$$

On the other hand, let $V\left(R^{2}\right)$ be a class of functions $\alpha$ of bounded variation over any finite rectangle for which

$$
\sup _{-\infty<m, n<\infty} \int_{n}^{n+1} \int_{m}^{m+1}\left|d \alpha\left(x_{1}, x_{2}\right)\right|<\infty \text {. }
$$

From now on, for the sake of simplicity, we shall use notations $x$, $u$ instead of $\left(x_{1}, x_{2}\right),\left(u_{1}, u_{2}\right)$ and also $f(x), \alpha(u)$ instead of $f\left(x_{1}, x_{2}\right), \alpha\left(u_{1}, u_{2}\right)$ and similarly in other cases.

Then for every pair of $f \in M\left(R^{2}\right)$ end $\alpha \in V\left(R^{2}\right)$, the Stieltjes integral of $f$ with respect to $\alpha$ is defined and denoted as follows:

$$
\int_{-\infty}^{\infty} \int_{-\infty}^{\infty} f(x-y) d \alpha(y) \quad\left(\forall x \in R^{2}\right)
$$

Let us also consider a path $\xi(t)$ in $R^{2}$ such as

$$
\xi(0)=0, \xi(t) \longrightarrow \infty \text { as } t \longrightarrow \infty \text {. }
$$

Then we have

THEOREM 3. Suppose $\alpha \in V\left(R^{2}\right)$, and for all $f \in M_{0}$,

$$
\lim _{t \rightarrow \infty} \int_{-\infty}^{\infty} \int_{-\infty}^{\infty} f(\xi(t)-\zeta-x) d \alpha(x)=A \int_{-\infty}^{\infty} \int_{-\infty}^{\infty} f(x) d x \quad\left(\forall \zeta \in R^{2}\right) .
$$

Then the limit relation

$$
\lim _{t \rightarrow \infty} \int_{-\infty}^{\infty} \int_{-\infty}^{\infty} g(\xi(t)-\zeta-x) d \alpha(x)=A \int_{-\infty}^{\infty} \int_{-\infty}^{\infty} g(x) d x \quad\left(\forall \zeta \in R^{2}\right)
$$

holds for every $g \in M\left(R^{2}\right)$.

Proof. Let us define for any $g \in M\left(R^{2}\right)$,

$$
a(x)=\int_{-\infty}^{\infty} \int_{-\infty}^{\infty} g(x-y) d \alpha(y) .
$$


Then we shall prove that $a(x) \in L^{\infty}\left(R^{2}\right)$ and is slowly oscillating along the path $\xi(t)$. First, we have

$$
\begin{aligned}
|a(x)| & =\left|\sum_{n=-\infty}^{\infty} \sum_{m=-\infty}^{\infty} \int_{n}^{n+1} \int_{m}^{m+1} g(x-y) d \alpha(y)\right| \\
& \leqq \sum_{n=-\infty}^{\infty} \sum_{m=-\infty}^{\infty} \max _{y \in I_{m, n}}|g(x-y)| \int_{n}^{n+1} \int_{m}^{m+1}|d \alpha(y)| \\
& \leqq 4 \sup _{-\infty<m, n<\infty} \int_{n}^{n+1} \int_{m}^{m+1}|d \alpha(y)| \sum_{n=-\infty}^{\infty} \sum_{m=-\infty}^{\infty} \max _{y \in I_{m, n}}|g(y)|<\infty,
\end{aligned}
$$

and second,

$$
\begin{aligned}
& |a(\xi(t)-\zeta)-a(x-\zeta)| \\
& \quad=\left|\int_{-\infty}^{\infty} \int_{-\infty}^{\infty}(g(\xi(t)-\zeta-y)-g(x-\zeta-y)) d \alpha(y)\right| \\
& \quad \leqq 4 \sup _{-\infty<m, n<\infty} \int_{n}^{n+1} \int_{m}^{m+1}|d \alpha(y)| \sum_{n=-\infty}^{\infty} \sum_{m=-\infty}^{\infty} \max _{y \in I_{m, n}}|g(\xi(t)-x+y)-g(y)|,
\end{aligned}
$$

since clearly $g(x)$ is uniformly continuous in any compact neighborhood of 0 , it will yield us

$$
|a(\xi(t)-\zeta)-a(x-\zeta)| \longrightarrow 0 \text { as } t \longrightarrow \infty \text { and } \xi(t)-x \longrightarrow 0\left(\forall \zeta \in R^{2}\right) \text {. }
$$

Now it follows that

$$
\begin{aligned}
\int_{-\infty}^{\infty} \int_{-\infty}^{\infty} f(\xi(t)-\zeta-x) a(x) d x \\
\quad=\int_{-\infty}^{\infty} \int_{-\infty}^{\infty} f(\xi(t)-\zeta-x) d x \int_{-\infty}^{\infty} \int_{-\infty}^{\infty} g(x-y) d \alpha(y) \\
\quad=\int_{-\infty}^{\infty} \int_{-\infty}^{\infty} d \alpha(y) \int_{-\infty}^{\infty} \int_{-\infty}^{\infty} f(\xi(t)-\zeta-x) g(x-y) d x \\
\quad=\int_{-\infty}^{\infty} \int_{-\infty}^{\infty} d \alpha(y) \int_{-\infty}^{\infty} \int_{-\infty}^{\infty} f(\xi(t)-\zeta-x-y) g(x) d x \\
=\int_{-\infty}^{\infty} \int_{-\infty}^{\infty} g(x) d x \int_{-\infty}^{\infty} \int_{-\infty}^{\infty} f(\xi(t)-\zeta-x-y) d \alpha(y)
\end{aligned}
$$

by the Fubini theorem and change of variables and again the Fubini theorem for the inversion of order of integration. By the Lebesgue convergence theorem with the formula (3.6),

$$
\int_{-\infty}^{\infty} \int_{-\infty}^{\infty} f(\xi(t)-\zeta-x) a(x) d x \longrightarrow A \int_{-\infty}^{\infty} \int_{-\infty}^{\infty} g(x) d x \int_{-\infty}^{\infty} \int_{-\infty}^{\infty} f(x) d x
$$

as $t \rightarrow \infty\left(\forall \zeta \in R^{2}\right)$. Hence we can apply Theorem $2^{\prime}$, to have 


$$
a(\xi(t)-\zeta) \longrightarrow A \int_{-\infty}^{\infty} \int_{-\infty}^{\infty} g(x) d x \text { as } t \longrightarrow \infty\left(\forall \zeta \in R^{2}\right)
$$

That is

$$
\int_{-\infty}^{\infty} \int_{-\infty}^{\infty} g(\xi(t)-\zeta-x) d \alpha(x) \longrightarrow A \int_{-\infty}^{\infty} \int_{-\infty}^{\infty} g(x) d x
$$

as $t \rightarrow \infty\left(\forall \zeta \in R^{2}\right)$. This completes the theorem.

We are going to apply this Tauberian theorem to prove the Wiener formula in the two-dimensional case. In fact, the Wiener formula we want to prove can be stated as the following theorem. For the sake of simplicity, we shall consider in the first quadrant. Let $R_{+}$be a half real line $(0, \infty)$ and $R_{+}^{2}$ be the first quadrant of the plane.

TheOREM 4. Suppose $f\left(x_{1}, x_{2}\right) \geqq 0$ in $\left(x_{1}, x_{2}\right) \in R_{+}^{2}$ and bounded on $R_{+}^{2}$. Then the limit relations

$$
\lim _{T \rightarrow \infty} \frac{1}{C_{1} C_{2} T^{2}} \int_{0}^{C_{2} T} \int_{0}^{C_{1} T} f\left(x_{1}, x_{2}\right) d x_{1} d x_{2}=A \quad\left(\forall C_{1}, C_{2}>0\right)
$$

and

$$
\lim _{\varepsilon \rightarrow 0} \frac{4 C_{1} C_{2}}{\pi^{2} \varepsilon^{2}} \int_{0}^{\infty} \int_{0}^{\infty} f\left(x_{1}, x_{2}\right) \frac{\sin ^{2} \varepsilon C_{1}^{-1} x_{1}}{x_{1}^{2}} \frac{\sin ^{2} \varepsilon C_{2}^{-1} x_{2}}{x_{2}^{2}} d x_{1} d x_{2}=A \quad\left(\forall C_{1}, C_{2}>0\right)
$$

are equivalent in the sense that if either of the limits (3.9) or (3.10) exists, then the other limit exists and assumes the same value.

Proof. Let us put

$$
x_{1}=e^{\xi_{1}}, \quad x_{2}=e^{\xi_{2}}
$$

and

$$
f\left(x_{1}, x_{2}\right)=\psi\left(\xi_{1}, \xi_{2}\right)
$$

So $\psi\left(\xi_{1}, \xi_{2}\right) \geqq 0$ in $\left(\xi_{1}, \xi_{2}\right) \in R^{2}$ and $\psi\left(\xi_{1}, \xi_{2}\right)$ is bounded on $R^{2}$. Again let us put

$$
C_{1}=e^{a}, \quad C_{2}=e^{b}
$$

and

$$
T=e^{5}, \quad \varepsilon=T^{-1} .
$$

Then (3.9) and (3.10) are written respectively by 


$$
\lim _{\zeta \rightarrow \infty} \int_{-\infty}^{\zeta+b} \int_{-\infty}^{\zeta+a} e^{-\left(\zeta+a-\xi_{1}\right)} e^{-\left(\zeta+b-\xi_{2}\right)} \psi\left(\xi_{1}, \xi_{2}\right) d \xi_{1} d \xi_{2}=A
$$

for all $(a, b) \in R^{2}$, and

$$
\lim _{\zeta \rightarrow \infty} \frac{4}{\pi^{2}} \int_{-\infty}^{\infty} \int_{-\infty}^{\infty} \frac{\sin ^{2} e^{-\left(\zeta+a-\xi_{1}\right)}}{e^{-\left(\zeta+a-\xi_{1}\right)}} \frac{\sin ^{2} e^{-\left(\zeta+b-\xi_{2}\right)}}{e^{-\left(\zeta+b-\xi_{2}\right)}} \psi\left(\xi_{1}, \xi_{2}\right) d \xi_{1} d \xi_{2}=A
$$

for all $(a, b) \in R^{2}$.

Since $\psi\left(\xi_{1}, \xi_{2}\right)$ is bounded on $R^{2}$,

$$
\sup _{-\infty<m, n<\infty} \int_{n}^{n+1} \int_{m}^{m+1} \psi\left(\xi_{1}, \xi_{2}\right) d \xi_{1} d \xi_{2}<\infty
$$

Now let us put

$$
g\left(\xi_{1}, \xi_{2}\right)=\int_{0}^{\xi_{2}} \int_{0}^{\xi_{1}} \psi\left(\eta_{1}, \eta_{2}\right) d \eta_{1} d \eta_{2}
$$

Then since $\psi\left(\eta_{1}, \eta_{2}\right) \geqq 0$,

$$
\begin{aligned}
\int_{n}^{n+1} \int_{m}^{m+1}\left|d g\left(\xi_{1}, \xi_{2}\right)\right| & =g(m+1, n+1)-g(m+1, n)-g(m, n+1)+g(m, x) \\
& =\int_{n}^{n+1} \int_{m}^{m+1} \psi\left(\xi_{1}, \xi_{2}\right) d \xi_{1} d \xi_{2},
\end{aligned}
$$

and so

$$
\sup _{-\infty<m, n<\infty} \int_{n}^{n+1} \int_{m}^{m+1}\left|d g\left(\xi_{1}, \xi_{2}\right)\right|<\infty
$$

that is, $g \in V\left(R^{2}\right)$.

Furthermore we can write (3.11) and (3.12) in the forms (3.15) and (3.17) respectively:

(3.15) $\lim _{\xi \rightarrow \infty} \int_{-\infty}^{\infty} \int_{-\infty}^{\infty} K_{1}\left(\zeta+a-\xi_{1}, \zeta+b-\xi_{2}\right) d g\left(\xi_{1}, \xi_{2}\right)=A \quad\left(\forall(a, b) \in R^{2}\right)$,

where

$$
K_{1}\left(\xi_{1}, \xi_{2}\right)= \begin{cases}e^{-\xi_{1}} e^{-\xi_{2}} & \left(\xi_{1}, \xi_{2}>0\right) \\ 0 & (\text { elsewhere }),\end{cases}
$$

and

(3.17) $\lim _{\xi \rightarrow \infty} \int_{-\infty}^{\infty} \int_{-\infty}^{\infty} K_{2}\left(\zeta+a-\xi_{1}, \zeta+b-\xi_{2}\right) d g\left(\xi_{1}, \xi_{2}\right)=A \quad\left(\forall(a, b) \in R^{2}\right)$, 
where

$$
K_{2}\left(\xi_{1}, \xi_{2}\right)=\frac{4}{\pi^{2}} \frac{\sin ^{2} e^{-\xi_{1}}}{e^{-\xi_{1}}} \frac{\sin ^{2} e^{-\xi_{2}}}{e^{-\xi_{2}}}
$$

Since

$$
\int_{-\infty}^{\infty} \int_{-\infty}^{\infty} K_{1}\left(\xi_{1}, \xi_{2}\right) d \xi_{1} d \xi_{2}=\int_{-\infty}^{\infty} \int_{-\infty}^{\infty} K_{2}\left(\xi_{1}, \xi_{2}\right) d \xi_{1} d \xi_{2}=1
$$

both (3.15) and (3.17) can also be written as follows:

(3.19) $\lim _{\zeta \rightarrow \infty} \int_{-\infty}^{\infty} \int_{-\infty}^{\infty} K_{1}\left(\zeta+a-\xi_{1}, \zeta+b-\xi_{2}\right) d g\left(\xi_{1}, \xi_{2}\right)=A \int_{-\infty}^{\infty} \int_{-\infty}^{\infty} K_{1}\left(\xi_{1}, \xi_{2}\right) d \xi_{1} d \xi_{2}$

$$
\left(\forall(a, b) \in R^{2}\right)
$$

and

(3.20) $\lim _{\zeta \rightarrow \infty} \int_{-\infty}^{\infty} \int_{-\infty}^{\infty} K_{2}\left(\zeta+a-\xi_{1}, \zeta+b-\xi_{2}\right) d g\left(\xi_{1}, \xi_{2}\right)=A \int_{-\infty}^{\infty} \int_{-\infty}^{\infty} K_{2}\left(\xi_{1}, \xi_{2}\right) d \xi_{1} d \xi_{2}$

$$
\left(\forall(a, b) \in R^{2}\right) \text {. }
$$

To show the equivalence of (3.15) and (3.17), we quote Theorem 3. The kernel $K_{2}$ clearly belongs to $M\left(R^{2}\right)$, since

$$
K_{2}\left(\xi_{1}, \xi_{2}\right)= \begin{cases}\frac{4}{\pi^{2}} e^{-\xi_{1}} e^{-\xi_{2}}\left(\frac{\sin e^{-\xi_{1}}}{e^{-\xi_{1}}}\right)^{2}\left(\frac{\sin e^{-\xi_{2}}}{e^{-\xi_{2}}}\right)^{2} & \left(0 \leqq \xi_{1}, \xi_{2}\right) \\ \frac{4}{\pi^{2}} e^{-\xi_{1}} e^{\xi_{2}}\left(\frac{\sin e^{-\xi_{1}}}{e^{-\xi_{1}}}\right)^{2} \sin ^{2} e^{-\xi_{2}} & \left(\xi_{2} \leqq 0 \leqq \xi_{1}\right) \\ \frac{4}{\pi^{2}} e^{\xi_{1}} e^{-\xi_{2}} \sin ^{2} e^{-\xi_{1}}\left(\frac{\sin e^{-\xi_{2}}}{e^{-\xi_{2}}}\right)^{2} & \left(\xi_{1} \leqq 0 \leqq \xi_{2}\right) \\ \frac{4}{\pi^{2}} e^{\xi_{1}} e^{\xi_{2}} \sin ^{2} e^{-\xi_{1}} \sin ^{2} e^{-\xi_{2}} & \left(\xi_{1}, \xi_{2} \leqq 0\right)\end{cases}
$$

and so

$$
\left|K_{2}\left(\xi_{1}, \xi_{2}\right)\right| \leqq \frac{4}{\pi^{2}} e^{-\left|\xi_{1}\right|} e^{-\left|\xi_{2}\right|}
$$

Its Fourier transform is

$$
\begin{aligned}
\hat{K}_{2}\left(u_{1}, u_{2}\right) & =\frac{1}{2 \pi} \prod_{k=1}^{2} \int_{-\infty}^{\infty} e^{\xi_{k}\left(1-i u_{k}\right)} \sin ^{2} e^{-\xi_{k}} d \xi_{k} \\
& =\frac{1}{2 \pi} \prod_{k=1}^{2} \frac{\Gamma\left(i u_{k}+1\right)}{u_{k}\left(i u_{k}-1\right)}\left(e^{-\pi u_{k} / 2}-e^{\pi u_{k} / 2}\right) 2^{-i u_{k}-1},
\end{aligned}
$$


and then $\hat{K}_{2}\left(u_{1}, u_{2}\right) \neq 0$ for any pair of real numbers $\left(u_{1}, u_{2}\right)$.

The kernel $K_{1}$ is discontinuous, and hence does not belong to $M\left(R^{2}\right)$. The modified kernels

$$
\begin{aligned}
K_{1}\left(\xi_{1}, \xi_{2} ; \varepsilon\right)= & \frac{1}{\varepsilon^{2}} \int_{\xi_{2}}^{\xi_{2}+\varepsilon} \int_{\xi_{1}}^{\xi_{1}+\varepsilon} K_{1}\left(\eta_{1}, \eta_{2}\right) d \eta_{1} d \eta_{2} \\
& = \begin{cases}e^{-\xi_{1}} \frac{1-e^{-\varepsilon}}{\varepsilon} \cdot e^{-\xi_{2}} \frac{1-e^{-\varepsilon}}{\varepsilon} & \left(0 \leqq \xi_{1}, \xi_{2}\right) \\
e^{-\xi_{1}} \frac{1-e^{-\varepsilon}}{\varepsilon} \cdot \frac{1-e^{-\xi_{2}-\varepsilon}}{\varepsilon} & \left(0 \leqq \xi_{1},-\varepsilon \leqq \xi_{2}<0\right) \\
\frac{1-e^{-\xi_{1}-\varepsilon}}{\varepsilon} \cdot e^{-\xi_{2}} \frac{1-e^{-\varepsilon}}{\varepsilon} & \left(-\varepsilon \leqq \xi_{1}<0,0 \leqq \xi_{2}\right) \\
\frac{1-e^{-\xi_{1}-\varepsilon}}{\varepsilon} \cdot \frac{1-e^{-\xi_{2}-\varepsilon}}{\varepsilon} & \left(-\varepsilon \leqq \xi_{1}, \xi_{2}<0\right) \\
0 & \text { (elsewhere) }\end{cases}
\end{aligned}
$$

do however belong to $M\left(R^{2}\right)$. Their Fourier transforms are

$$
\begin{aligned}
\hat{K}_{1}\left(u_{1},\right. & \left.u_{2} ; \varepsilon\right) \\
& =\frac{1}{2 \pi} \int_{-\infty}^{\infty} \int_{-\infty}^{\infty} e^{-i\left(u_{1} \xi_{1}+u_{2} \xi_{2}\right)} d \xi_{1} d \xi_{2} \frac{1}{\varepsilon^{2}} \int_{\xi_{2}}^{\xi_{2}+\varepsilon} \int_{\xi_{1}}^{\xi_{1}+\varepsilon} K_{1}\left(\eta_{1}, \eta_{2}\right) d \eta_{1} d \eta_{2} \\
& =\frac{1}{2 \pi} \int_{-\infty}^{\infty} \int_{-\infty}^{\infty} e^{-i\left(u_{1} \xi_{1}+u_{2} \xi_{2}\right)} d \xi_{1} d \xi_{2} \frac{1}{\varepsilon^{2}} \int_{0}^{e} \int_{0}^{\varepsilon} K_{1}\left(\xi_{1}+\eta_{1}, \xi_{2}+\eta_{2}\right) d \eta_{1} d \eta_{2} \\
& =\prod_{k=1}^{2} \frac{1}{\varepsilon \sqrt{2 \pi}} \int_{0}^{s} d \eta_{k} \int_{-\eta_{k}}^{\infty} e^{-\xi_{k}\left(1+i u_{k}\right)-\eta_{k}} d \xi_{k} \\
& =\prod_{k=1}^{2} \frac{1}{\varepsilon \sqrt{2 \pi}} \int_{0}^{\varepsilon} \frac{e^{i u_{k} \eta_{k}}}{1+i u_{k}} d \eta_{k} \\
& =\prod_{k=1}^{2} \frac{1}{\varepsilon \sqrt{2 \pi}} \frac{e^{i u_{k} \varepsilon}-1}{i u_{k}\left(1+i u_{k}\right)} .
\end{aligned}
$$

Thus $\hat{K}_{1}\left(u_{1}, u_{2} ; \varepsilon\right)=0$ only for $u_{1}=2 m \pi / \varepsilon, u_{2}=2 n \pi / \varepsilon(m, n=0, \pm 1, \pm 2, \cdots)$. Hence there is no one $\left(u_{1}, u_{2}\right)$ for which this vanishes for every $\varepsilon$. As for detailed calculations, refer to the original paper of $N$. Wiener [10] or N. Wiener [11]. Now we shall consider the subclass $M_{0}=\left\{K_{1}\left(\xi_{1}, \xi_{2} ; \varepsilon\right)\right.$ : $\varepsilon>0\}$ instead of $K_{1}\left(\xi_{1}, \xi_{2}\right)$. Since

$$
\int_{-\infty}^{\infty} \int_{-\infty}^{\infty} K_{1}\left(\xi_{1}, \xi_{2} ; \varepsilon\right) d \xi_{1} d \xi_{2}=1 \quad(\forall \varepsilon>0),
$$

we can apply Theorem 3 and the equivalence of (3.17) and 


$$
\lim _{\xi \rightarrow \infty} \int_{-\infty}^{\infty} \int_{-\infty}^{\infty} K_{1}\left(\zeta+a-\xi_{1}, \zeta+b-\xi_{2} ; \varepsilon\right) d g\left(\xi_{1}, \xi_{2}\right)=A
$$

$$
\left(\forall(a, b) \in R^{2}, \forall \varepsilon>0\right)
$$

are established.

Finally, we shall prove the equivalence of (3.15) and (3.21). First, we assume (3.15). Let us notice that

$$
\begin{aligned}
\int_{-\infty}^{\infty} \int_{-\infty}^{\infty} K_{1}\left(\zeta+a-\xi_{1}, \zeta+b-\xi_{2} ; \varepsilon\right) d g\left(\xi_{1}, \xi_{2}\right) \\
\quad=\int_{-\infty}^{\infty} \int_{-\infty}^{\infty}\left\{\frac{1}{\varepsilon^{2}} \int_{\zeta+b-\xi_{2}}^{\zeta+b-\xi_{2}+\varepsilon} \int_{\zeta+a-\xi_{1}}^{\zeta+a-\xi_{1}+\varepsilon} K_{1}\left(\eta_{1}, \eta_{2}\right) d \eta_{1} d \eta_{2}\right\} d g\left(\xi_{1}, \xi_{2}\right) \\
=\int_{-\infty}^{\infty} \int_{-\infty}^{\infty}\left\{\frac{1}{\varepsilon^{2}} \int_{0}^{s} \int_{0}^{\varepsilon} K_{1}\left(\zeta+a-\xi_{1}+\eta_{1}, \zeta+b-\xi_{2}+\eta_{2}\right) d \eta_{1} d \eta_{2}\right\} d g\left(\xi_{1}, \xi_{2}\right) \\
=\frac{1}{\varepsilon^{2}} \int_{0}^{\varepsilon} \int_{0}^{e} d \eta_{1} d \eta_{2}\left\{\int_{-\infty}^{\infty} \int_{-\infty}^{\infty} K_{1}\left(\zeta+a+\eta_{1}-\xi_{1}, \zeta+b+\eta_{2}-\xi_{2}\right) d g\left(\xi_{1}, \xi_{2}\right)\right\} .
\end{aligned}
$$

Applying the Lebesgue convergence theorem, it follows that

$$
\begin{aligned}
\lim _{\varepsilon \rightarrow \infty} \int_{-\infty}^{\infty} \int_{-\infty}^{\infty} K_{1}\left(\zeta+a-\xi_{1}, \zeta+b-\xi_{2} ; \varepsilon\right) d g\left(\xi_{1}, \xi_{2}\right) \\
=\frac{1}{\varepsilon^{2}} \int_{0}^{\varepsilon} \int_{0}^{\varepsilon} A d \eta_{1} d \eta_{2}=A \quad\left(\forall(a, b) \in R^{2}, \forall \varepsilon>0\right),
\end{aligned}
$$

which is (3.21). Second, we assume (3.21). Let us notice that

$$
\left(\frac{1-e^{-\varepsilon}}{\varepsilon}\right)^{2} K_{1}\left(\xi_{1}, \xi_{2}\right) \leqq K_{1}\left(\xi_{1}, \xi_{2} ; \varepsilon\right) \leqq\left(\frac{e^{\varepsilon}-1}{\varepsilon}\right)^{2} K_{1}\left(\xi_{1}+\varepsilon, \xi_{2}+\varepsilon\right) \text {. }
$$

Since $g\left(\xi_{1}, \xi_{2}\right)$ is defined by (3.14), we then see

$$
\begin{aligned}
& \left(\frac{1-e^{-\varepsilon}}{\varepsilon}\right)^{2} \varlimsup_{\zeta \rightarrow \infty} \int_{-\infty}^{\infty} \int_{-\infty}^{\infty} K_{1}\left(\zeta+a-\xi_{1}, \zeta+b-\xi_{2}\right) d g\left(\xi_{1}, \xi_{2}\right) \\
& \leqq \varlimsup_{\zeta \rightarrow \infty} \int_{-\infty}^{\infty} \int_{-\infty}^{\infty} K_{1}\left(\zeta+a-\xi_{1}, \zeta+b-\xi_{2} ; \varepsilon\right) d g\left(\xi_{1}, \xi_{2}\right) \\
& \leqq\left(\frac{e^{\varepsilon}-1}{\varepsilon}\right)^{2} \lim _{\zeta \rightarrow \infty} \int_{-\infty}^{\infty} \int_{-\infty}^{\infty} K_{1}\left(\zeta+a-\xi_{1}+\varepsilon, \zeta+b-\xi_{2}+\varepsilon\right) d g\left(\xi_{1}, \xi_{2}\right) \\
& \leqq\left(\frac{e^{\varepsilon}-1}{\varepsilon}\right)^{2} \frac{\lim }{\zeta \rightarrow \infty} \int_{-\infty}^{\infty} \int_{-\infty}^{\infty} K_{1}\left(\zeta+a-\xi_{1}, \zeta+b-\xi_{2}\right) d g\left(\xi_{1}, \xi_{2}\right),
\end{aligned}
$$

and so

$$
\left(\frac{1-e^{-\varepsilon}}{\varepsilon}\right)^{2} \varlimsup_{\varepsilon \rightarrow \infty} \int_{-\infty}^{\infty} \int_{-\infty}^{\infty} K_{1}\left(\zeta+a-\xi_{1}, \zeta+b-\xi_{2}\right) d g\left(\xi_{1}, \xi_{2}\right)
$$




$$
\leqq A \leqq\left(\frac{e^{\varepsilon}-1}{\varepsilon}\right)^{2} \frac{\lim }{\zeta \rightarrow \infty} \int_{-\infty}^{\infty} \int_{-\infty}^{\infty} K_{1}\left(\zeta+a-\xi_{1}, \zeta+b-\xi_{2}\right) d g\left(\xi_{1}, \xi_{2}\right) .
$$

As $\varepsilon$ is arbitrarily small, we may make $\left(1-e^{-\varepsilon}\right) / \varepsilon$ and $\left(e^{\varepsilon}-1\right) / \varepsilon$ as close to 1 as we wish, and hence

$$
\begin{aligned}
& \varlimsup_{\zeta \rightarrow \infty} \int_{-\infty}^{\infty} \int_{-\infty}^{\infty} K_{1}\left(\zeta+a-\xi_{1}, \zeta+b-\xi_{2}\right) d g\left(\xi_{1}, \xi_{2}\right) \\
& \quad \leqq A \leqq \lim _{\zeta \rightarrow \infty} \int_{-\infty}^{\infty} \int_{-\infty}^{\infty} K_{1}\left(\zeta+a-\xi_{1}, \zeta+b-\xi_{2}\right) d g\left(\xi_{1}, \xi_{2}\right) \quad\left(\forall(a, b) \in R^{2}\right),
\end{aligned}
$$

which is equivalent to (3.15). This completes the proof of Theorem 4.

REMARK. In Theorem 4, we have only used the condition of boundedness of function $f$ to show

$$
\sup _{-\infty<m, n<\infty} \int_{n}^{n+1} \int_{m}^{m+1} \psi\left(\xi_{1}, \xi_{2}\right) d \xi_{1} d \xi_{2}<\infty .
$$

Therefore we can replace the condition of boundedness by somewhat weaker one.

(i) If $f \in L_{10 \mathrm{c}}^{1}\left(R_{+}^{2}\right)$ and

$$
\sup _{-\infty<m, n<\infty} \int_{e^{n}}^{e^{n+1}} \int_{e^{m}}^{e^{m+1}} \frac{f\left(x_{1}, x_{2}\right)}{x_{1} x_{2}} d x_{1} d x_{2}<\infty,
$$

then (3.13) is equivalent to (3.22).

(ii) If $f \in L_{10 c}^{1}\left(R_{+}^{2}\right)$ and

$$
\begin{aligned}
& \int_{1}^{T} \int_{0}^{1} f\left(x_{1}, x_{2}\right) d x_{1} d x_{2}=O(T) \text { as } \quad T \longrightarrow \infty, \\
& \int_{0}^{1} \int_{1}^{s} f\left(x_{1}, x_{2}\right) d x_{1} d x_{2}=O(S) \text { as } S \longrightarrow \infty,
\end{aligned}
$$

and

$$
\sup _{0 \leqq m, n<\infty} \int_{e^{n}}^{e^{n+1}} \int_{e^{m}}^{e^{m+1}} \frac{f\left(x_{1}, x_{2}\right)}{x_{1} x_{2}} d x_{1} d x_{2}<\infty,
$$

then (3.13) is also true.

In Theorem 4 , it is clear that we can take $f\left(x_{1}, x_{2}\right)=0$ if $0 \leqq x_{1} \leqq 1$ and $0 \leqq x_{2} \leqq 1$ without loss of generality.

First, we assume (3.9), (3.23) and (3.24). Then we can suppose that $f\left(x_{1}, x_{2}\right)=0$ if either $0 \leqq x_{1} \leqq 1$ or $0 \leqq x_{2} \leqq 1$ by (3.23) and (3.24), and therefore the condition (3.22) of (i) is replaced by (3.25).

Second, we assume (3.10), (3.23) and (3.24). Then we have 


$$
\lim _{\varepsilon \rightarrow 0} \frac{4 C_{1} C_{2}}{\pi^{2} \varepsilon^{2}} \int_{1}^{\infty} \int_{0}^{1} f\left(x_{1}, x_{2}\right) \frac{\sin ^{2} \varepsilon C_{1}^{-1} x_{1}}{x_{1}^{2}} \frac{\sin ^{2} \varepsilon C_{2}^{-1} x_{2}}{x_{2}^{2}} d x_{1} d x_{2}=0 \quad\left(\forall C_{1}, C_{2}>0\right)
$$

and

$$
\lim _{x \rightarrow 0} \frac{4 C_{1} C_{2}}{\pi^{2} \varepsilon^{2}} \int_{0}^{1} \int_{1}^{\infty} f\left(x_{1}, x_{2}\right) \frac{\sin ^{2} \varepsilon C_{1}^{-1} x_{1}}{x_{1}^{2}} \frac{\sin ^{2} \varepsilon C_{2}^{-1} x_{2}}{x_{2}^{2}} d x_{1} d x_{2}=0
$$

$$
\left(\forall C_{1}, C_{2}>0\right) \text {. }
$$

Because, we have by $f\left(x_{1}, x_{2}\right) \geqq 0$,

$$
\begin{aligned}
0 & \leqq \varlimsup_{\varepsilon \rightarrow 0} \frac{4 C_{1} C_{2}}{\pi^{2} \varepsilon^{2}} \int_{1}^{\infty} \int_{0}^{1} f\left(x_{1}, x_{2}\right) \frac{\sin ^{2} \varepsilon C_{1}^{-1} x_{1}}{x_{1}^{2}} \frac{\sin ^{2} \varepsilon C_{2}^{-1} x_{2}}{x_{2}^{2}} d x_{1} d x_{2} \\
& =\varlimsup_{\varepsilon \rightarrow 0} \frac{4 C_{2}}{\pi^{2} C_{1}} \int_{1}^{\infty}\left\{\int_{0}^{1} f\left(x_{1}, x_{2}\right)\left(\frac{\sin _{1} \varepsilon C_{1}^{-1} x_{1}}{\varepsilon C_{1}^{-1} x_{1}}\right)^{2} d x_{1}\right\} \frac{\sin ^{2} \varepsilon C_{2}^{-1} x_{2}}{x_{2}^{2}} d x_{2} \\
& \leqq \varlimsup_{\varepsilon \rightarrow 0} \frac{2 \varepsilon}{\pi C_{1}} \cdot \frac{2 C_{2}}{\pi \varepsilon} \int_{1}^{\infty}\left\{\int_{0}^{1} f\left(x_{1}, x_{2}\right) d x_{1}\right\} \frac{\sin ^{2} \varepsilon C_{2}^{-1} x_{2}}{x_{2}^{2}} d x_{2} .
\end{aligned}
$$

Now if we apply the one-sided Wiener formula of S. Koizumi [3], the last formula does not exceed

$$
\varlimsup_{\varepsilon \rightarrow 0} O(\varepsilon) \cdot \varlimsup_{T \rightarrow \infty} \int_{1}^{T}\left\{\int_{0}^{1} f\left(x_{1}, x_{2}\right) d x_{1}\right\} d x_{2},
$$

which turns out to be $o(1)$ as $\varepsilon \rightarrow 0$ by (3.23). Thus we have (3.26). Similarly, we have (3.27) by (3.24). Therefore we can also suppose that $f\left(x_{1}, x_{2}\right)=0$ if either $0 \leqq x_{1} \leqq 1$ or $0 \leqq x_{2} \leqq 1$, and the condition (3.22) of (i) is replaced by (3.25).

(iii) If $f \in L_{\mathrm{loc}}^{1}\left(R_{+}^{2}\right)$ and

$$
\frac{1}{S T} \int_{0}^{T} \int_{0}^{S} f\left(x_{1}, x_{2}\right) d x_{1} d x_{2} \leqq B
$$

for any $S, T>0$, where $B$ is a constant, then (3.13) is also true.

Since $f\left(x_{1}, x_{2}\right) \geqq 0$, we have for any pair of integers $(m, n)$

$$
\begin{aligned}
\int_{e^{n}}^{e^{n+1}} & \int_{e^{m}}^{e^{m+1}} \frac{f\left(x_{1}, x_{2}\right)}{x_{1} x_{2}} d x_{1} d x_{2} \\
& \leqq e^{2} \cdot \frac{1}{e^{m+1} e^{n+1}} \int_{0}^{e^{n+1}} \int_{0}^{e m+1} f\left(x_{1}, x_{2}\right) d x_{1} d x_{2} \leqq e^{2} B
\end{aligned}
$$

by (3.28) and it follows (3.22) of (i). It is noticed that (3.28) also implies the conditions (3.23) and (3.24) of (ii).

Instead of the condition of boundedness, there is another kind of 
condition to make (3.13) hold.

(iv) If $f \in L_{1 \mathrm{oc}}^{1}\left(R_{+}^{2}\right)$ and the limit of either (3.9) or (3.10) exists uniformly as for $C_{1}, C_{2}>0$, then'(3.13) is also true.

First, we assume the uniform existence of (3.9) as for $C_{1}, C_{2}>0$. Since $\psi\left(\xi_{1}, \xi_{2}\right) \geqq 0$, it follows for all sufficiently large values of $\zeta$,

$$
\begin{aligned}
A+1 & >\int_{-\infty}^{\zeta+b} \int_{-\infty}^{\zeta+a} e^{-\left(\zeta+a-\xi_{1}\right)} e^{-\left(\zeta+b-\xi_{2}\right)} \psi\left(\xi_{1}, \xi_{2}\right) d \xi_{1} d \xi_{2} \\
& \geqq \int_{\zeta+b-1}^{\zeta+b} \int_{\zeta+a-1}^{\zeta+a} e^{-\left(\zeta+a-\xi_{1}\right)} e^{-\left(\zeta+b-\xi_{2}\right)} \psi\left(\xi_{1}, \xi_{2}\right) d \xi_{1} d \xi_{2} \\
& \geqq e^{-2} \int_{\zeta+b-1}^{\zeta+b} \int_{\zeta+a-1}^{\zeta+a} \psi\left(\xi_{1}, \xi_{2}\right) d \xi_{1} d \xi_{2} \quad\left(\forall(a, b) \in R^{2}\right) .
\end{aligned}
$$

Now we take one of sufficiently large $\zeta$ and fix it, and if we choose $(a, b)$ such that $\zeta+a-1=m, \zeta+b-1=n$ for any pair of integers $(m, n)$, then we have by the above estimation

$$
A+1 \geqq e^{-2} \int_{n}^{n+1} \int_{m}^{m+1} \psi\left(\xi_{1}, \xi_{2}\right) d \xi_{1} d \xi_{2} .
$$

Thus we have (3.13).

Second, we assume the uniform existence of (3.10) as for $C_{1}, C_{2}>0$. It follows for all sufficiently large values of $\zeta$,

$$
\begin{aligned}
A+1> & \frac{4}{\pi^{2}} \int_{-\infty}^{\infty} \int_{-\infty}^{\infty} \frac{\sin ^{2} e^{-\left(\zeta+a-\xi_{1}\right)}}{e^{-\left(\zeta+a-\xi_{1}\right)}} \frac{\sin ^{2} e^{-\left(\zeta+b-\xi_{2}\right)}}{e^{-\left(\zeta+b-\xi_{2}\right)}} \psi\left(\xi_{1}, \xi_{2}\right) d \xi_{1} d \xi_{2} \\
\geqq & \frac{4}{\pi^{2}} \int_{\zeta+b}^{\zeta+b+1} \int_{\zeta+a}^{\zeta+a+1} e^{-\left(\zeta+a-\xi_{1}\right)} e^{-\left(\zeta+b-\xi_{2}\right)} \\
& \cdot\left(\frac{\sin e^{-\left(\zeta+a-\xi_{1}\right)}}{e^{-\left(\zeta+a-\xi_{1}\right)}}\right)^{2}\left(\frac{\sin e^{-\left(\zeta+b-\xi_{2}\right)}}{e^{-\left(\zeta+b-\xi_{2}\right)}}\right)^{2} \psi\left(\xi_{1}, \xi_{2}\right) d \xi_{1} d \xi_{2} \\
\geqq & \frac{4}{\pi^{2}}\left(\frac{\sin e}{e}\right)^{4} \int_{\zeta+b}^{\zeta+b+1} \int_{\zeta+a}^{\zeta+a+1} \psi\left(\xi_{1}, \xi_{2}\right) d \xi_{1} d \xi_{2} \quad\left(\forall(a, b) \in R^{2}\right) .
\end{aligned}
$$

We similarly have (3.13).

\section{References}

[1] T. Kawata, Fourier analysis of nonstationary stochastic processes, Trans. Amer. Math. Soc., 118 (1965), 276-302.

[2] S. KorzuMr, On the Hilbert transform I, J. Fac. Sci. Hokkaido Univ., Series I, 14 (1959), 153-224.

[3] S. KoIzUMI, Hilbert transform in the Stepanoff space, Proc. Japan Acad., 38 (1962), 735-740.

[4] K. Matsuora, Generalized harmonic analysis of functions of two variables, Keio Engineering Reports, 33 (1980), 67-81. 
[5] K. Matsuora, On the generalized Hilbert transforms of functions of two variables, Keio Engineering Reports, 33 (1980), 97-116.

[6] K. Matsuoka, Some theorems about almost periodic functions of two variables, to appear.

[7] H. R. Pitr, General Tauberian theorems, Proc. London Math. Soc., 44 (1938), 243-288.

[8] W. RUdis, Fourier Analysis on Groups, Interscience Tracts in Pure and Applied Math., No. 12, Interscience Publishers, New York, 1960.

[9] N. WiEner, Generalized harmonic analysis, Acta, Math., 55 (1930), 117-258.

[10] N. WiEner, Tauberian theorems, Ann. of Math., 33 (1932), 1-100.

[11] N. WiEner, The Fourier Integral and Certain of its Applications, Cambridge Univ. Press, London, 1933.

Present Address:

Department of Mathematics

KAGAWA UNIVERSITY

SaIWAi-Cho, Takamatsu, 760

Department of Mathematics

KEIO UNIVERSITY

HIYOSHI-CHO, КоноRU-RU, YоRонама, 223

AND

DEPartMent OF Mathematics

KEIO UNIVERSITY

НIYOSHI-Сно, КоноKU-KU, ҮоконамA, 223 